\title{
Rho GTPase CDC42 regulates directionality and random movement via distinct MAPK pathways in neutrophils
}

\author{
Kathleen Szczur, Haiming Xu, Simon Atkinson, Yi Zheng, and Marie-Dominique Filippi
}

\begin{abstract}
Neutrophil transmigration into tissue is a multiple-step process that results from a coordinated rearrangement of the cytoskeleton and adhesion complexes. Assembly and disassembly of actin and adhesion structures dictate motility behavior, while polarity and gradient sensing provide directionality to the cell movement. Here, using mice deficient in the CDC42 regulator CDC42 GTPase-activating protein (CDC42GAP), we demonstrate that CDC42 activity separately regulates neutrophil motility and directionality.
\end{abstract}

ing edge, while CDC42GAP-induced p38 ${ }^{\mathrm{MAPK}}$ phosphorylation regulates directed migration by antagonizing filopodia assembly. Overall, this study reveals that CDC42 activity regulates both motility and directionality in neutrophils, but via distinct mitogen-activated protein kinase (MAPK) pathways. (Blood. 2006; 108:4205-4213)

๑ 2006 by The American Society of Hematology

\section{Introduction}

Neutrophils are critical in the inflammatory process. The recruitment of neutrophils to sites of inflammation requires a series of highly regulated adhesive and chemotactic events. These cellular activities result from the activation of signals from various receptors, including adhesion molecules such as selectins and integrins, and chemokine receptors. Failure to regulate any of these events may lead to abnormal innate immune responses, including immunodeficiency or aberrant inflammatory reactions. Although the process of neutrophil extravasation has been well studied, the intracellular molecular events that ultimately regulate these processes still remain to be understood in detail, particularly in physiologic settings.

Cell migration is a multiple-step process that results from a coordinated rearrangement of the cytoskeleton and adhesion complexes. ${ }^{1}$ Upon chemoattractant stimulation, cells polarize and form lamellipodia containing $\mathrm{F}$-actin at the leading edge oriented toward the source of stimulation and a contractile uropod. The lamellipodia are stabilized to the substratum by the assembly of adhesion complexes, which mature into focal adhesions. These complexes serve as traction forces necessary for the translocation of the cell body. Completion of cell migration requires actin disruption to allow both adhesion complex turnover at the leading edge and retraction of the tail. Thus, the cytoskeleton, through assembly and disassembly of F-actin and adhesion structures, is believed to dictate motility behavior, while polarity and gradient sensing give direction to the cell movement.

The key integrators of signals emanating from chemokine receptors and integrin molecules that coordinate these processes are members of the small Rho GTPase family, including Rac,
CDC42, and Rho. ${ }^{2}$ Rho GTPases cycle between inactive GDPbound and active GTP-bound forms. Guanine nucleotide exchange factors (GEFs) promote the activation of GTPases by stimulating the exchange of GDP to GTP, while GTPase-activating proteins (GAPs) accelerate hydrolysis of GTP, returning the GTPase to an inactive form. ${ }^{3}$ Activation of CDC42 controls the formation of filopodia and is critical for gradient sensing. ${ }^{4}$ Rac induces membrane protrusion via lamellipodia. ${ }^{4}$ In addition, both Rac and CDC42 regulate the formation of focal complexes that stabilize the leading edge of the cell. Activation of Rho generates contractility via stress fiber and focal adhesions. ${ }^{4,5}$ The details of the regulation of cell migration are highly cell-type specific. The use of genetargeted mice provides an approach to study the role of Rho GTPases in primary cells in a lineage-specific fashion and has provided important information regarding the physiologic function of these proteins in multiple blood cell types. ${ }^{6-16}$

CDC42 is a well-known regulator of cell chemotaxis. In the neutrophil cell line HL60, or in macrophages, CDC42 inhibitors prevent cells from maintaining polarity and a persistent leading edge. ${ }^{17,18}$ In addition, CDC42 inhibitors induce formation of multiple short leading edges containing filamentous actin. ${ }^{18}$ These observations suggest that CDC42 activity is required to properly orient the cytoskeleton responses with respect to the direction of the chemoattractant. Using a mouse genetically deficient in the CDC42 GEF PIX $\alpha$, which leads to loss of CDC42 activity, Li et $\mathrm{al}^{19}$ demonstrated that, upon activation of G-coupled receptors, free $\mathrm{G} \beta$ binds to $\mathrm{p} 21$-activated kinase (PAK), which then interacts with PIX $\alpha$. This binding stimulates CDC42 activity and, in turn, leads to activation of PAK. The PIX $\alpha / \mathrm{PAK} / \mathrm{CDC} 42$ axis is essential for
From the Department of Experimental Hematology, Cincinnati Children's Research Foundation, University Cincinnati College of Medicine, $\mathrm{OH}$; and Department of Medicine, Indiana University School of Medicine, Indianapolis, IN.

Submitted March 30, 2006; accepted August 7, 2006. Prepublished online as Blood First Edition Paper, August 24, 2006; ZDOI 10.1182/blood-2006-03-013789.
The online version of this manuscript contains a data supplement.

The publication costs of this article were defrayed in part by page charge payment. Therefore, and solely to indicate this fact, this article is hereby marked "advertisement" in accordance with 18 USC section 1734.

C 2006 by The American Society of Hematology 
direction sensing and persistent polarized migration toward the source of stimulation by stimulating assembly of F-actin at the leading edge of the cell and by regulating the localization of an antagonist of F-actin assembly, PTEN, at the rear of the cell. ${ }^{19}$ Thus, CDC42 activity serves as a gradient sensor during neutrophil chemotaxis. The best-studied CDC42 effectors are Wiskott-Aldrich syndrome protein (WASp) and PAK, both of which are known to be involved in cytoskeleton reorganization. ${ }^{4,20}$ Although considerable progress has been made in understanding the role of CDC42 in physiologic settings, very little is known about the role of CDC42 upon integrin engagement during neutrophil migration and the associated adhesive structures or the consequences of a hyperactivity of CDC42 in these cells.

We have recently demonstrated that mice deficient in the CDC42 regulator CDC42GAP, which represents the gain of CDC42 activity, display defects in hematopoietic stem cell migration, adhesion, and F-actin reorganization. These changes were associated with abnormal erythropoiesis. ${ }^{21}$ In the present study, we examined the consequences of gain of CDC42 activity on neutrophil migration and provide evidence that CDC42 activity is important during inflammatory responses downstream of integrin molecules by regulating both random movement and directed migration via distinct mitogen-activated protein kinase (MAPK) signaling pathways.

\section{Materials and methods}

\section{Generation of animal model}

Cells from day-14.5 fetal liver CDC42GAP ${ }^{-1-}$ mice $^{21}$ and wild-type (WT) littermates were transplanted into lethally irradiated C57BL/6 recipients (1175 cGy in a split dose; Jackson Laboratories, Bar Harbor, ME). Animals were used for experiments 5 weeks after bone marrow reconstitution. All animals were bred in the Cincinnati Children's Research Foundation pathogen-free animal facility. All experimental procedures were approved by the institutional animal committee.

\section{Neutrophil migration in vivo}

Mice were challenged with $3 \%$ thioglycollate (Sigma, St Louis, MO) by intraperitoneal injection $(1 \mathrm{~mL})$. Peritoneal lavages were performed 4 and 18 hours after challenge. ${ }^{6}$ Cells were counted by hemocytometer. Neutrophil content was evaluated after cytospin preparation of the cells and Diff Quick staining (Dade Berhing, Deerfield, IL).

\section{Neutrophil isolation}

Neutrophils were isolated from bone marrow cells by percoll gradient as previously described. ${ }^{6}$ Neutrophils were also generated after culture of low-density bone marrow cells as previously described. ${ }^{12,15}$ Neutrophil purity was similar between genotypes and estimated between $65 \%$ and $75 \%$ depending on experiments. No differences in the phenotype of the cells were observed between the 2 methods of neutrophil isolation.

\section{Neutrophil migration assays in vitro}

Neutrophil migration was evaluated in triplicate using a 48-well microchemotaxis chamber (Neuro Probe, Cabin John, MD) as described previously. ${ }^{6,12,15}$ In the chemotaxis assay, fMLP $(1 \mu \mathrm{M}$; Sigma) is added in the lower chamber. In the chemokinesis assay, fMLP is added in the upper chamber with the cells and in the lower chamber. Numbers of migrated cells were determined by counting 3 randomly chosen fields.

Migration was also evaluated using 3- $\mu \mathrm{m}$ transwell chambers (Corning Inc, Corning, NY) coated with fibrinogen $(25 \mu \mathrm{g} / \mathrm{mL}$; Sigma) in chemokinesis and chemotaxis assays. The cells $\left(4 \times 10^{5}\right)$ were diluted in $100 \mu \mathrm{L}$ Hanks balanced salt solution (HBSS; Invitrogen, Carlsbad, CA), $1 \mathrm{mM}$
$\mathrm{Ca}^{2+}$, and $1 \mathrm{mM} \mathrm{Mg}^{2+}$ with or without $10 \mu \mathrm{M}$ fMLP, and migration toward fMLP was allowed for 3 hours. The migrated cells in the bottom well were counted with a hemocytometer.

To examine neutrophil transendothelial migration, human umbilical vein endothelial cells (HUVECs) $\left(4 \times 10^{3}\right)$ were seeded in the upper chamber of the transwell, and the cells were grown for 4 days at $37^{\circ} \mathrm{C}$ until confluence. Neutrophils $\left(5 \times 10^{5}\right)$ in $100 \mu \mathrm{L} \mathrm{HBSS}, 1 \mathrm{mM} \mathrm{Ca}^{2+}$, and $1 \mathrm{mM}$ $\mathrm{Mg}^{2+}$ with or without $10 \mu \mathrm{M}$ fMLP were allowed to migrate toward fMLP for 3 hours. The migrated cells in the bottom well were counted with a hemocytometer.

Time-lapse video microscopy was performed in a Zigmond chamber (Neuro Probe, Gaithersburg, MD). After adhesion onto glass cover slips, the cells were mounted onto the Zigmond chamber. Migration was allowed in a gradient of $10 \mu \mathrm{M}$ fMLP diluted in HBSS, $1 \%$ gelatin, and 1mM HEPES (pH 7.6) for 30 minutes. Microscope images were recorded at 55 interval with a Zeiss microscope (Oberkochen, Germany) at $10 \times / 0.3$ NA oil objective magnification equipped with an ORCA-ER camera (Hamamatsu, Tokyo, Japan) and driven by Openlab software (Improvision, Lexington, MA). A translocation rate of 23 to 25 freshly isolated neutrophils, shown in Figure 2, or 50 neutrophils generated by in vitro culture (not shown) from each genotype and from independent videos were analyzed using Openlab.

\section{Neutrophil adhesion assay}

Neutrophils $\left(2 \times 10^{5}\right)$ were allowed to adhere to fibrinogen $(25 \mu \mathrm{g} / \mathrm{mL})$ for 30 minutes in HBSS, $1 \mathrm{mM} \mathrm{Ca}^{2+}$, and $1 \mathrm{mM} \mathrm{Mg}^{2+}$ with or without $10 \mu \mathrm{M}$ fMLP at $37^{\circ} \mathrm{C}$, as previously described. ${ }^{12}$

\section{Integrin receptor expression}

To determine $\beta 2$-integrin expression upon stimulation, neutrophils $\left(5 \times 10^{5}\right)$ were incubated with $10 \mu \mathrm{M}$ fMLP for 30 minutes at $37^{\circ} \mathrm{C}$. The cells were washed with $\mathrm{HBSS}$ at $4^{\circ} \mathrm{C}$, and stained with phycoerythrin (PE)-labeled antimouse antibodies against Mac-1 (CD11b, M1/70) or CD18 (C71/16) (Pharmingen, San Diego, CA) at $4^{\circ} \mathrm{C}$. Integrin expression was analyzed by flow cytometry using Facscanto (Becton Dickinson, Mountain View, CA). Fluorescence intensity is reported as median channel fluorescence (MCF) in arbitrary units.

\section{Immunofluorescence}

To characterize F-actin assembly and adhesion structures upon integrin ligation, neutrophils $\left(5 \times 10^{4}\right)$ were prestimulated with $10 \mu \mathrm{M}$ fMLP in HBSS, $1 \mathrm{mM} \mathrm{Ca}^{2+}$, and $1 \mathrm{mM} \mathrm{Mg}^{2+}$ and seeded onto fibrinogen-coated slides for 30 minutes at $37^{\circ} \mathrm{C}$. The cells were then fixed with $2 \%$ paraformaldehyde and stained with rhodamine-labeled phalloidin (Molecular Probes, Eugene, OR) or mouse antivinculin (V-11-5; Sigma) followed by anti-mouse Alexa488 (Molecular Probes). A Z series of fluorescence images was captured with a Leica DMIRB fluorescence microscope (Wetzlar, Germany) at $63 \times 10.70$ NA magnification (air objective) and an ORCA-ER C4742-95 camera (Hamamatsu) equipped with a deconvolution system (Leica, CA) driven by Openlab software. ${ }^{12,15}$ The $\mathrm{Z}$ series was analyzed by deconvolution using Volocity (Improvision). Images were acquired with Openlab 4.0.3 (Improvision) and analyzed using Volocity (Improvision).

\section{MAPK activities}

Neutrophils $\left(1-2 \times 10^{6}\right)$ were first stimulated with $10 \mu \mathrm{M}$ fMLP for 0,1 , and 5 minutes at $37^{\circ} \mathrm{C}$. The cells were lysed in Triton-based buffer and analyzed for phospho-p42/p44 $4^{\mathrm{MAPK}}$ and phospho-p38 $8^{\mathrm{MAPK}}$ by immunoblot. ${ }^{12}$ The membranes were stripped and reprobed with $\mathrm{p} 42 / \mathrm{p} 44^{\mathrm{MAPK}}$ and p38 $8^{\mathrm{MAPK}}$ for loading controls (all antibodies [Abs] were from Cell Signaling, Beverly, MA).

To assess MAPK activities upon integrin ligation, the cells $\left(14 \times 10^{6}\right)$ were resuspended in HBSS, $1 \mathrm{mM} \mathrm{Ca}^{2+}$, and $1 \mathrm{mM} \mathrm{Mg}^{2+}$ with $10 \mu \mathrm{M}$ fMLP, and seeded onto fibrinogen-coated plates for 5, 15, and 30 minutes. The nonadherent cells were removed and the adherent cells were recovered from the plates using cold cell-dissociation buffer. The cells were lysed and analyzed for MAPK activities as described in the paragraph above. 
To determine the role of extracellular signal-regulated kinase ${ }^{\mathrm{MAPK}}$ $\left(\mathrm{ERK}^{\mathrm{MAPK}}\right)$ and $\mathrm{p} 38^{\mathrm{MAPK}}$ in neutrophil migration, neutrophils were preincubated with 2 to $10 \mu \mathrm{M}$ of the MAPK kinase (MEK) inhibitor U0126 (Calbiochem, San Diego, CA) or $10 \mu \mathrm{M}$ of the p38 $8^{\mathrm{MAPK}}$ inhibitor SB203580 (Calbiochem) or $10 \mu \mathrm{M}$ DMSO for 30 minutes at $37^{\circ} \mathrm{C}$. Migration assays and F-actin structure analysis were performed as described under "Neutrophil migration assays in vitro" and "Immunofluorescence" with similar inhibitor concentration.

\section{Rho GTPase activity}

Neutrophils were stimulated on fibrinogen-coated plates and $10 \mu \mathrm{M}$ fMLP in HBSS, $\mathrm{Ca}^{2+}$, and $\mathrm{Mg}^{2+}$ for 10 minutes. The cells were lysed onto plates with $\mathrm{Mg}^{2+}$-based lysis buffer and assessed for Rac and CDC42 pull-down assay using PAK-PBD (Upstate, Charlottesville, VA) or for RhoA using Rhotekin-PBD (Upstate), as previously described. ${ }^{12}$ Total cell lysates were analyzed for CDC42, Rac, or RhoA expression with anti-CDC42 or anti-Rac (Transduction Laboratories, San Diego, CA), or anti-RhoA (Santa Cruz Biotechnology, Santa Cruz, CA) as a loading control.

\section{Results}

\section{Gain of CDC42 activity: animal model}

CDC42GAP-deficient mice have been previously described. ${ }^{21}$ Due to perinatal lethality in most $\mathrm{CDC} 42 \mathrm{GAP}^{-1-}$ mice, day-14.5 $\mathrm{CDC}_{2} \mathrm{GAP}^{-1-}$ fetal livers were used to reconstitute hematopoiesis in irradiated congenic WT animals. Complete donor chimerism was demonstrated in these mice (not shown). CDC42GAP protein was barely detectable in neutrophils derived from the bone marrow of mice reconstituted with $\mathrm{CDC} 42 \mathrm{GAP}^{-/-}$cells by immunoblot (data not shown), similar to that of CDC42GAP-deficient animals. ${ }^{21}$ In addition, CDC42GAP ${ }^{-1-}$ neutrophils demonstrated a significant increase in GTP-bound CDC42 compared with that of WT without interfering with Rac and RhoA activities (Figure 1A), which is consistent with CDC42GAP-deficient animals. ${ }^{21}$ Mice reconstituted with $\mathrm{CDC}_{2} \mathrm{GAP}^{-1-}$ cells did not show any abnormalities in peripheral blood or bone marrow neutrophil numbers or in bone marrow-derived neutrophil differentiation in vitro (data not shown). Therefore, mice reconstituted with $\mathrm{CDC}_{2} \mathrm{GAP}^{-1-}$ hematopoietic cells represent an animal model of hematopoietic-specific gain of CDC42 activity.

\section{The loss of CDC42GAP function is associated with increased neutrophil recruitment during inflammation}

To address the role of CDC42 activity in neutrophils during inflammation, we elicited neutrophils into the peritoneal cavity 4 and 18 hours after thioglycollate instillation. ${ }^{6}$ The number of neutrophils recruited into the peritoneal cavities of $\mathrm{CDC}_{2} \mathrm{GAP}^{-1-}$ reconstituted mice after challenge was slightly but significantly higher than WT controls at both time points (Figure 1B). Thus, deregulation of CDC42 activity leads to modest increased neutrophil response to inflammatory stimulus in vivo.

\section{The loss of CDC42GAP function is associated with increased motility, while directed migration is defective}

To dissect the role of CDC42GAP in neutrophil migration, we next examined the migration of $\mathrm{CDC}_{2} \mathrm{GAP}^{-/-}$neutrophils using time-lapse videomicroscopy. In response to the fMLP gradient, WT cells polarized with a single membrane extension that rapidly oriented toward the agonist gradient. Migration appeared coordinated with membrane extension at the leading edge and tail retraction oriented in a straight line, allowing the cells to efficiently

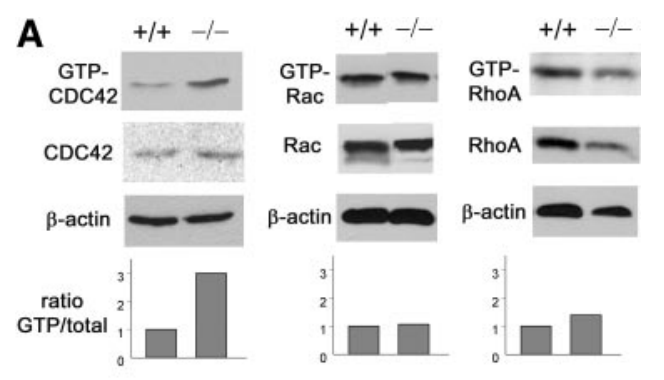

B

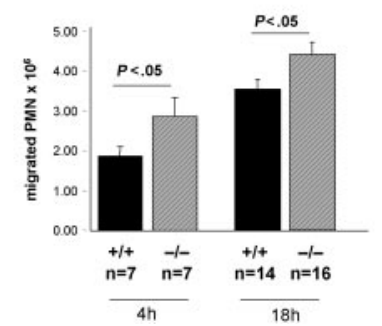

Figure 1. Model of gain of CDC42 activity. C57BL/6 cells mice reconstituted with WT or CDC42GAP ${ }^{-1-}$ embryonic day $(\mathrm{E})-14.5$ fetal liver cells. The reconstituted animals were used 5 weeks after bone marrow reconstitution. (A) CDC42, Rac, and RhoA activities of bone marrow-derived neutrophils. Rho GTPase activity was assessed by the standard pull-down assay using the PAK-binding domain for CDC42 and Rac or the Rhotekin-binding domain for RhoA. The histogram represents the relative ratio of GTP-Rho GTPase versus total protein. $\beta$-actin was used as loading control. (B) Neutrophil recruitment into peritoneal cavities after challenge with $3 \%$ thioglycollate. Peritoneal lavages were performed 4 and 18 hours after challenge, and total cells were enumerated with a hemocytometer. Neutrophil content was evaluated after cytospin preparation of the cells and Diff Quick staining. Mean \pm SEM from 3 independent experiments.

translocate toward the source of stimulation (Figure 2A; see also Video S1, available on the Blood website by clicking on the Supplemental Videos link at the top of the online article). In contrast, CDC42GAP ${ }^{-1-}$ neutrophils showed multiple surface extensions in various directions and abnormal filopodia in the tail in a subset of cells (Videos S2-S3; Figure 2A-B). In addition, the altered leading edge was accompanied by a change in cell direction and, as a result, a significant number of $\mathrm{CDC}_{2} \mathrm{GAP}^{-1-}$ cells migrated in a direction away from the agonist gradient (Video S2 and Figure 2A,C). The speed of migration of $\mathrm{CDC}_{2} 2 \mathrm{GAP}^{-1-}$ cells that successfully oriented toward the fMLP gradient was significantly faster than that of WT cells (Figure 2D). Therefore, proper regulation of $\mathrm{CDC} 42$ activity appears to be important in vitro to maintain an appropriate direction toward the agonist gradient and to regulate the velocity of cell migration.

To further understand the role of CDC42 activity in neutrophil migration, we quantified migration in vitro using Boyden chamber assays. Chemokinesis was assessed in a uniform concentration of fMLP in both wells of the chamber, while chemotaxis was assessed in a gradient in which fMLP was placed only in the lower chamber. The number of WT cells that migrated in response to fMLP during chemotaxis was significantly higher than the number of neutrophils exhibiting fMLP-induced chemokinesis (Figure 3A). In contrast, the numbers of migrated $\mathrm{CDC} 42 \mathrm{GAP}^{-1-}$ neutrophils were similar in both assays (Figure 3A). This difference was due both to an increased $\mathrm{CDC} 42 \mathrm{GAP}^{-1-}$ neutrophil chemokinesis, and a significantly diminished $\mathrm{CDC}_{2} \mathrm{GAP}^{-/-}$neutrophil chemotaxis compared with that of WT controls.

Neutrophils transmigrate across the endothelial barrier. To better mimic a transmigration process, fMLP-stimulated neutrophil migration was examined in transwells precoated with fibrinogen, a major $\beta 2$-integrin ligand. In this assay, $\mathrm{CDC} 42 \mathrm{GAP}^{-/-}$neutrophils demonstrated increased number of migrated cells during both 
A
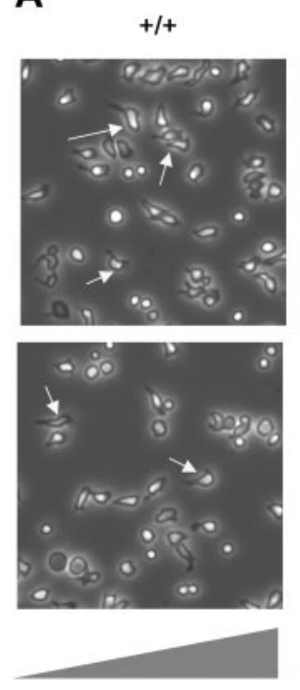

fMLP
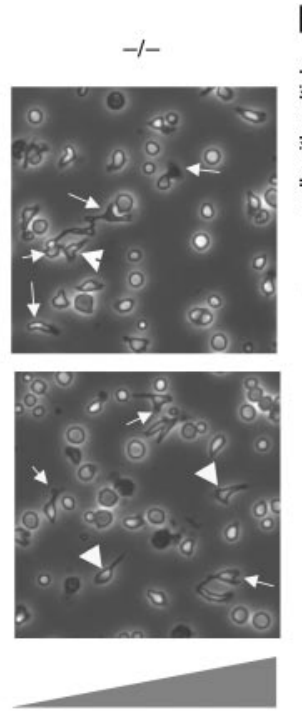

fMLP
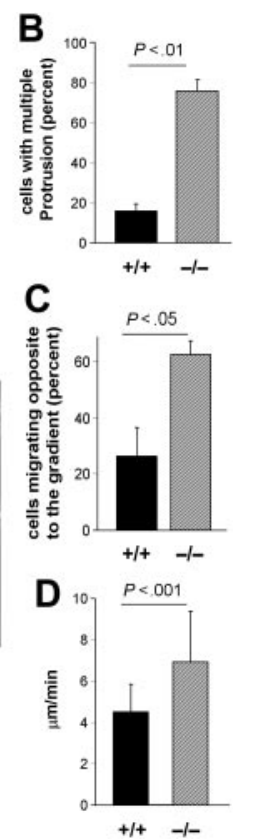

Figure 2. Loss of CDC42GAP expression is associated with increased motility while directed migration is defective. Neutrophil migration was examined by time-lapse video microscopy in a gradient of $10 \mu \mathrm{M}$ fMLP in a Zigmond chamber. (A) Representative images of cells during the course of migration; fMLP concentration increases from left to right. Videos S1-S3 have more representative images. WT cells orient and migrate toward the source of fMLP (arrows). CDC42GAP ${ }^{-1-}$ cells displayed abnormal membrane protrusion (arrows) and some cells migrated away from the gradient (arrowheads). (B) The percentage of cells displaying more than one membrane protrusion was enumerated. (C) The percentage of cells that migrated away from high fMLP concentration is indicated in the histogram. (D) The average translocation rate was measured using Openlab. The result from freshly isolated neutrophils is shown. Similar results were obtained from neutrophils generated in in vitro culture. All histograms represent the mean $\pm S D ; n=23$ to 25 cells from 2 to 3 independent videos.

fMLP-induced chemokinesis and chemotaxis compared with that of WT cells (Figure 3B). This observation is consistent with an increased motility in $\mathrm{CDC} 42 \mathrm{GAP}^{-1-}$ neutrophils compared with that of WT. In addition, WT cells but not $\mathrm{CDC}_{2} \mathrm{GAP}^{-1-}$ cells demonstrated significantly more migrated neutrophils in chemotaxis versus chemokinesis (Figure $3 \mathrm{~B}$ ). This observation is also consistent with a defective directed migration in $\mathrm{CDC}_{2} \mathrm{GAP}^{-1-}$ cells compared with WT cells. Similar results were obtained using a transendothelial migration assay across HUVECs (Figure 3C), reinforcing the physiologic relevance of CDC42-mediated neutrophil migration. Of note, in transwell assays, despite an apparent defective directed migration, more $\mathrm{CDC} 42 \mathrm{GAP}^{-1-}$ cells migrated during chemotaxis than did the WT, which is likely due to the overall increased motility associated with CDC42GAP deficiency. This effect was not observed in the Boyden chamber assay. The apparent difference between the Boyden chamber and the transwell assays may reflect distinct integrin dependency of the assays to support cell migration. Indeed, migration is a complex process which requires coordination of signals from both chemokine and integrin receptors to control both chemokinesis and chemotaxis responses. Despite an apparent lack of specific substratum, migration measured in Boyden or Zigmond chambers also requires integrin engagement. However, these various assays may engage distinct integrins. Since migration can be differentially regulated depending on specific integrins, ${ }^{22}$ a change in integrin engagement could change the level of migration and therefore account for different level of cell migration measured by the Boyden chamber and transwell assays.

To determine whether the abnormal migration of $\mathrm{CDC} 42 \mathrm{GAP}^{-/-}$ neutrophils was related to abnormal adhesion, we analyzed adhesion to fibrinogen. Adhesion to fibrinogen was similar between each genotype (Figure 3D). In addition, CD18 integrin was expressed in $\mathrm{CDC}_{2} \mathrm{GAP}^{-1-}$ cells at a level similar to that in WT cells, both before and after stimulation with fMLP (Figure 3E). These data indicate that the abnormal migration of $\mathrm{CDC}_{2} \mathrm{GAP}^{-1-}$ cells was not due to altered adhesion or integrin receptor expression.

These results reflect an overall increased cell motility in $\mathrm{CDC}_{2} \mathrm{GAP}^{-/-}$neutrophils while directed migration is defective, suggesting a role for CDC42GAP in both random movement and directed migration in neutrophils in physiologically relevant in vitro models downstream of chemokine and/or integrin receptors.

The loss of CDC42GAP function is associated with abnormal assembly of filopodia and F-actin-containing podosomelike structures

Migration requires a coordinated rearrangement of the cytoskeleton and associated adhesion structures. ${ }^{1}$ To determine whether altered CDC42 activity effected cytoskeleton rearrangement, F-actin reorganization was examined in neutrophils prestimulated with fMLP on fibrinogen-coated slides. In WT cells, integrin ligation induced formation of a lamellipodium at the leading edge, accompanied in some cells by a punctuated pattern of F-actin located behind the cell's leading edge (Figure 4A). Some WT neutrophils also demonstrated the formation of filopodia concentrated at the leading edge (not shown). Consistent with the increased membrane protrusions observed by time-lapse microscopy, a significant number of $\mathrm{CDC}_{2} \mathrm{GAP}^{-/-}$cells displayed increased filopodia-like formations
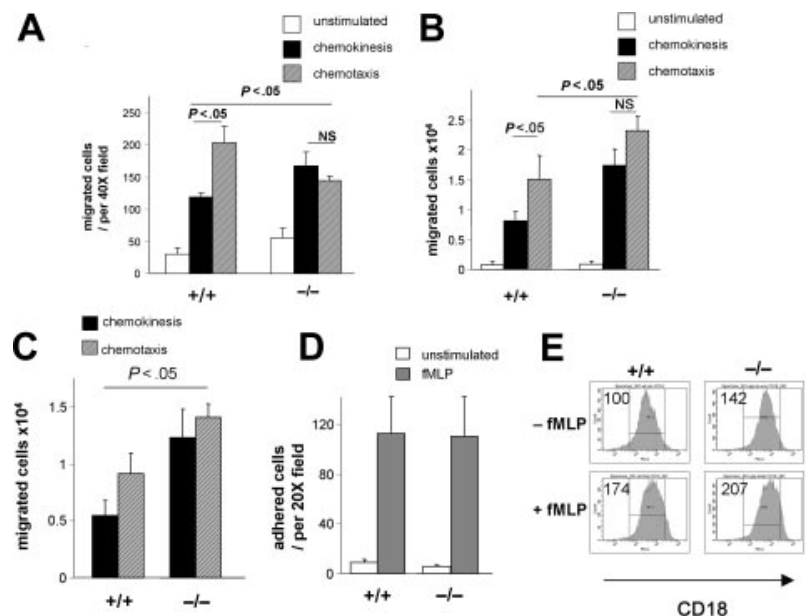

Figure 3. CDC42GAP regulates both random movement and directed migration. (A) Neutrophil migration using the Boyden chamber. Migration was evaluated without stimulation, in uniform concentration, or in a gradient of $1 \mu \mathrm{M}$ fMLP to measure chemokinesis or chemotaxis, respectively. The histogram represents the number of migrated neutrophils per field, mean $\pm \mathrm{SD}$; representative experiment in triplicate of 3 independent experiments is shown. (B) Neutrophil migration using transwells coated with fibrinogen. Migration was evaluated without $\mathrm{MLP}$ or in uniform concentration or in a gradient of $10 \mu \mathrm{M}$ fMLP. The histogram represents the total number of migrated neutrophils recovered from the bottom well, mean $\pm S D$; representative experiment in triplicate from 3 independent experiments is shown $(n=9)$. (C) Neutrophil migration was evaluated using transendothelial migration across HUVECs in uniform concentration or in a gradient of $10 \mu \mathrm{M}$ fMLP. The histogram represents the total number of migrated neutrophils recovered from the bottom well, mean $\pm S D$; representative experiment in triplicate from 3 independent experiments is shown. (D) Adhesion to fibrinogen. Neutrophils were allowed to adhere to fibrinogen for 30 minutes in the absence or in the presence of $10 \mu \mathrm{M}$ fMLP. Histogram represents the number of adherent cells counted per field, mean $\pm \mathrm{SD}$; representative experiment in triplicate of 3 independent experiments is shown. (E) $\beta-2$ integrin expression as assessed by flow cytometry before and after stimulation with $10 \mu \mathrm{M}$ fMLP. The numbers indicate the relative median channel fluorescence. 
A
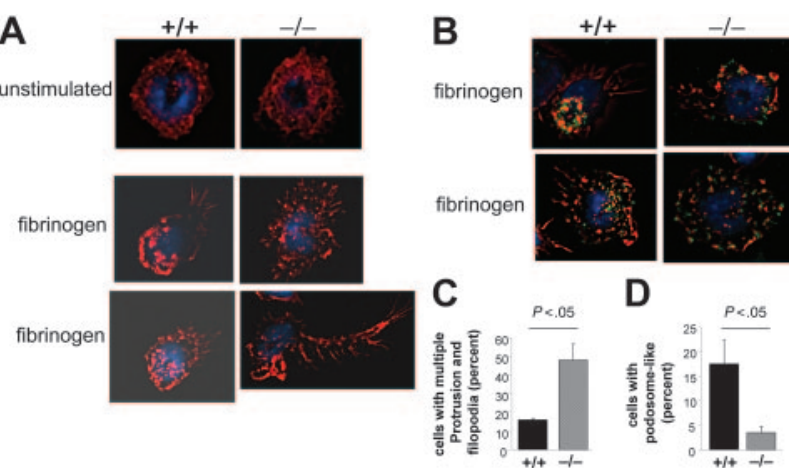

Figure 4. Loss of CDC42GAP functions is associated with abnormal assembly of podosome-like structures and filopodia. F-actin assembly and organization of adhesion structures. (A) Neutrophils were prestimulated with $10 \mu \mathrm{M}$ fMLP and seeded on fibrinogen-coated slides for 30 minutes. The cells were then fixed and stained with rhodamine-phalloidin (in red). Figure S1 shows more representative pictures. Two representative images of each genotype from 3 independent experiments are shown. (B) Neutrophils were prestimulated with FMLP and seeded on fibrinogen-coated slides for 30 minutes. The cells were then fixed and stained with rhodamine-phalloidin (in red) and antivinculin followed by anti-mouse Alexa488 (in green). Two representative images of each genotype from 3 independent experiments are shown. (C) The percentage of cells displaying more than 1 membrane protrusion and increased filopodia was quantified. (D) The percentage of cells displaying podosome-like structures was quantified. All histograms represent the mean \pm SD from 3 independent experiments.

all around the cell (Figure 4A,C), including the tail (Figure S1). A subset of $\mathrm{CDC} 42 \mathrm{GAP}^{-/-}$neutrophils demonstrated multiple "heads" or leading edges (Figure S1). Finally, the punctuate organization of F-actin behind the leading edge of WT cells was absent in a significant number of CDC42GAP ${ }^{-1-}$ cells (Figure 4A).

Because the punctuate pattern of F-actin was reminiscent of podosomes seen in macrophages and osteoclasts, WT cells were stained for vinculin-associated adhesion structures. In WT cells, punctuate staining of F-actin was surrounded by a ring of vinculin (Figure 4B). These podosome-like structures were, in general, organized in clusters behind the cell's leading edge. In some WT cells, podosome-like structures assembled in a ring-like pattern (Figure 4B). However, in contrast with other cell types that display podosomes, under our experimental conditions the formation of a peripheral belt of podosomes was not observed in neutrophils. In CDC42GAP ${ }^{-1-}$ cells, vinculin was located at the periphery of the cells (Figure 4B). Some CDC42GAP ${ }^{-1-}$ cells displayed F-actin associated with vinculin in a dispersed manner in the cells (Figure $4 \mathrm{~B}$ ). The number of $\mathrm{CDC}_{2} \mathrm{GAP}^{-1-}$ neutrophils displaying podosome-like structures at the leading edges of cells was significantly reduced compared with WT cells (Figure 4B,D). These results indicate that CDC42 activity regulates filopodia formation and a cell-adhesion structure in neutrophils that morphologically resembles podosomes.

\section{CDC42GAP regulates MAPK signaling in neutrophils}

The MAPK family has been largely implicated in the regulation of cell migration. ${ }^{23}$ ERK ${ }^{\mathrm{MAPK}}$ regulates focal adhesion turnover and velocity of migration in fibroblasts, ${ }^{24-27}$ while $\mathrm{p} 38^{\mathrm{MAPK}}$ inhibits filopodia formation and stimulates chemotaxis. ${ }^{28}$ To dissect the signaling pathways by which CDC42 activity regulates neutrophil migration and to determine whether CDC42GAP acts downstream of integrin versus chemokine receptors, we studied the activity of these MAPKs in $\mathrm{CDC}_{42 \mathrm{GAP}^{-/-}}$neutrophils after integrin ligation and fMLP stimulation or after fMLP stimulation alone. Upon integrin ligation, ERK activity peaked at 5 minutes both in WT and $\mathrm{CDC} 4 \mathrm{GAP}^{-1-}$ neutrophils. In WT cells, ERK phosphorylation was transient, and the level of ERK activity returned to near baseline after 15 minutes. CDC42GAP ${ }^{-1-}$ cells demonstrated significantly higher levels of phosphorylated ERK for up to 30 minutes compared with that of WT controls (Figure 5A). In contrast, the amount of phosphorylated $\mathrm{p} 38^{\mathrm{MAPK}}$ was decreased

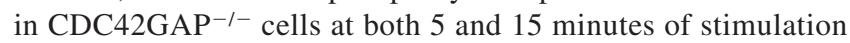
compared with that in WT cells (Figure 5B). Abnormal MAPK activities associated with CDC42GAP deficiency were not observed when the cells were stimulated with fMLP alone (Figure 5C). Thus, these results suggest that CDC42GAP integrates these MAPK activities downstream of integrin receptors combined with chemokine receptors and that integrins can mediate signals via CDC42GAP for both random movement and directed migration.

CDC42GAP-mediated ERK activity regulates chemokinesis and podosome-like assembly, while CDC42GAP-mediated p38 ${ }^{\text {MAPK }}$ activity regulates chemotaxis and restrains filopodialike formation

To further understand the roles of ERK and $\mathrm{p} 38^{\mathrm{MAPK}}$ in CDC42GAPmediated neutrophil migration, we treated WT and CDC42GAP ${ }^{-1-}$ cells with pharmacologic inhibitors of MEK and p38 $8^{\mathrm{MAPK}}$. We analyzed fMLP-induced chemokinesis and chemotaxis in Boyden chambers and transwells coated with fibrinogen.

We first examined whether the increased chemokinesis in CDC42GAP-deficient cells was associated with high ERK or low p3 $8^{\mathrm{MAPK}}$ activities in these cells. In the Boyden chamber assay, $\mathrm{CDC}_{2} \mathrm{GAP}^{-/-}$cells treated with U0126, a MEK inhibitor, demonstrated neutrophil chemokinesis similar to that of WT cells (Figure 6A). In contrast, treatment with the p38 $8^{\mathrm{MAPK}}$ inhibitor SB203580 did not alter chemokinesis of WT cells or the abnormal chemokinesis responses of $\mathrm{CDC}^{2} \mathrm{GAP}^{-/-}$cells (Figure 6A). U0126 treatment in $\mathrm{CDC}_{42} \mathrm{GAP}^{-1-}$ cells also rescued increased chemokinesis of CDC42GAP ${ }^{-1-}$ cells to WT levels in fibrinogen-coated transwells (Figure 6B). In this assay, SB203580 treatment did not increase
A

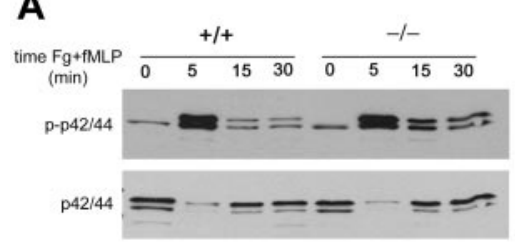

B

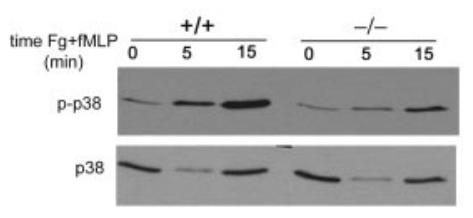

C

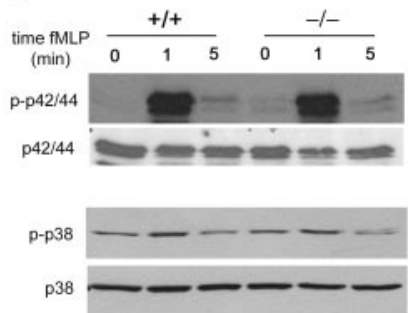

Figure 5. CDC42GAP regulates MAPK signaling in neutrophils. (A-B) Neutrophils were prestimulated with fMLP and seeded on fibrinogen for the indicated time. The cells were lysed and analyzed for phosphorylated p42/44 followed by total p42/44 (A) and for phosphorylated p38 followed by total p38 (B). (C) Neutrophils were stimulated with fMLP alone for the indicated time and examined for phosphorylated p42/44 and phosphorylated p38. Representative blot from 2 to 3 independent experiments is shown. 
A

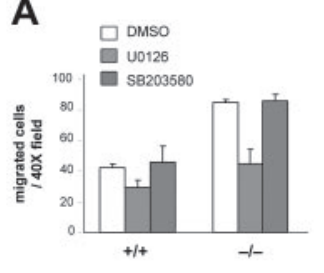

D

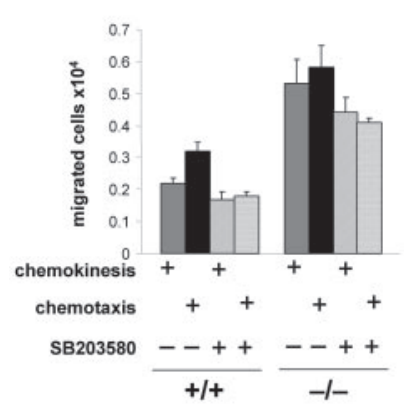

B

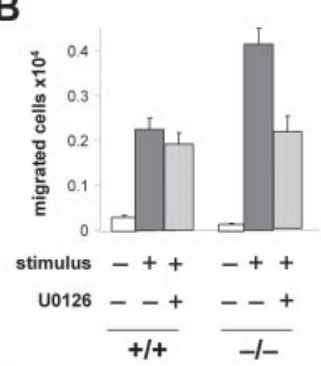

E
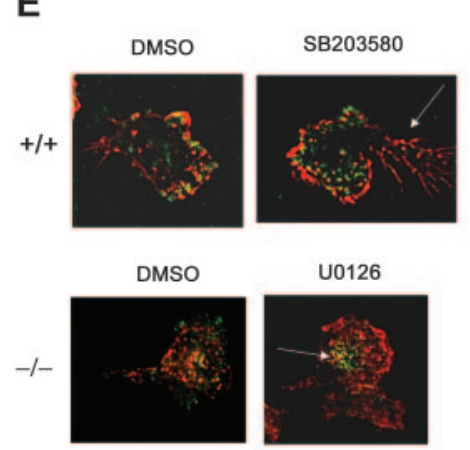

C

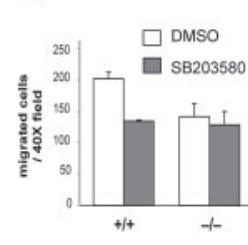

$\mathbf{F}$

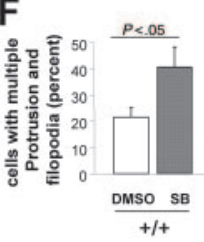

G

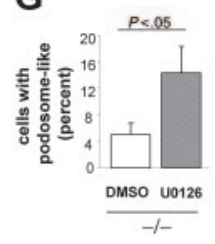

Figure 6. CDC42GAP-mediated ERK activity regulates chemokinesis and podosome-like assembly, while CDC42GAP-mediated $\mathrm{p} 38^{\mathrm{MAPK}}$ activity regulates chemotaxis and restrains filopodia-like formation. Neutrophils were treated with either the MEK inhibitor U0126 or the p38MAPK inhibitor SB203580 and subjected to migration assays or F-actin and vinculin reorganization. (A) Chemokinesis assessed in the Boyden chamber as in Figure $3 A$. (B) Chemokinesis in the presence of U0126 assessed in transwells coated with fibrinogen as in Figure 3B. (C) Chemotaxis in the presence of SB203580 assessed in the Boyden chamber. (D) Chemokinesis and chemotaxis in the presence of SB203580 assessed in transwells coated with fibrinogen. All histograms represent the mean $\pm \mathrm{SD}$; a representative experiment in triplicate from 3 independent experiments is shown. (E) Neutrophils were prestimulated and seeded on fibrinogen for 30 minutes and examined for F-actin assembly and vinculin structures as in Figure 4B. Note the presence of filopodia in the tail in WT cells treated with SB203580 (arrow). Note the presence of podosome-like structures at the leading edge of the cells in CDC42GAP-deficient cells treated with U0126 (arrow). Representative images from 3 independent experiments are shown. $(F)$ The percentage of WT cells treated with SB203580 displaying more than 1 membrane protrusion and increased filopodia was quantified. (G) The percentage of CDC42GAP-deficient cells treated with U0126 displaying podosome-like structures was quantified. Histograms represent the mean \pm SD of 3 independent experiments. chemokinesis responses of WT cells (Figure 6D). These data indicate that high ERK activity but not low p38 $8^{\mathrm{MAPK}}$ activity in CDC42GAP-deficient cells correlates with increased chemokinesis and suggest that the ERK but not the p38 $8^{\mathrm{MAPK}}$ pathway may regulate CDC42GAP-mediated chemokinesis.

We next examined the potential role of $\mathrm{p} 38^{\mathrm{MAPK}}$ in CDC42mediated chemotaxis. In the Boyden chamber assay, the number of migrated cells in SB203580-treated WT cells was similar to that of $\mathrm{CDC}_{42} \mathrm{GAP}^{-1-}$ cells (Figure $6 \mathrm{C}$ ). In the transwell assay, SB203580-treated WT cells showed diminished chemotaxis (Figure 6D). SB203580 treatment of CDC42GAP ${ }^{-1-}$ cells did not change the overall increased migration of these cells compared with that of WT neutrophils, although the level of cell migration of both WT and CDC42 $\mathrm{GAP}^{-1-}$ cells was diminished compared with that of the same cells treated with the vehicle control. Overall, these results indicate that low $\mathrm{p} 38^{\mathrm{MAPK}}$ activity in CDC42GAP-deficient cells correlates with defective directed migration and suggest that $\mathrm{p} 38^{\mathrm{MAPK}}$ may regulate chemotaxis mediated by CDC42GAP.

Finally, to correlate CDC42GAP-mediated migration and MAPK activities with cytoskeleton and adhesion structure reorganization, cells treated with the inhibitors were analyzed for F-actin and vinculin-associated adhesion structures. Clusters of podosome-like structures were rescued in $\mathrm{CDC}_{2} \mathrm{GAP}^{-/-}$cells treated with $\mathrm{U} 0126$ (Figure 6E,G), suggesting that high ERK activity in CDC42GAPdeficient cells correlates with the absence of these structures. In addition, WT cells treated with SB203580 developed filopodia-like structures in the tail (Figure 6E-F), and some cells displayed multiple leading edges (not shown). These changes were similar to the phenotype of $\mathrm{CDC}_{2} \mathrm{GAP}^{-1-}$ cells. However, the formation of clusters of podosome-like structures was not abrogated in WT cells by SB203580 treatment. These results suggest that low p38 ${ }^{\mathrm{MAPK}}$ activity in CDC42GAP-deficient cells correlates with increased filopodia-like formation but not the absence of podosome-like structures.

In conclusion, this study suggests that regulation of CDC42 activity via CDC42GAP regulates both random movement and directed migration, but via distinct MAPK signals. CDC42 activity appears to mediate neutrophil chemokinesis via ERK-dependent podosome-like structures, while CDC42-dependent directed migration may require $\mathrm{p} 38^{\mathrm{MAPK}}$ signals to antagonize lateral membrane protrusion and filopodia formation.

\section{Discussion}

Migration is a multiple-step process regulated by Rho GTPases. ${ }^{1,2}$ Upon stimulation, cells polarize and form a lamellipodium of F-actin at the leading edge, which is stabilized to the substratum by adhesion complexes. These complexes mature into focal adhesions, which give the contractile forces necessary for cell translocation. Completion of cell movement requires adhesion turnover at the leading edge and retraction of the tail. Thus, assembly and disassembly of adhesion structures dictate motility behavior, while polarity and gradient sensing give the direction of the cell movement. Using mice deficient in the CDC42 regulator CDC42GAP, we present evidence that, in primary neutrophils, CDC42 activity plays a physiologic and important role in both motility and directionality via distinct MAPK signaling pathways.

We and others have suggested a role for CDC42 activity in cell motility. In various cell lineages, ${ }^{17,29,30}$ CDC42 activity has been associated with increased the velocity of nondirectional migration. ${ }^{17,29,30}$ Our data confirm and extend these previous reports. First, our study highlights the physiologic relevance of CDC42 activity in neutrophil motility, since the increased neutrophil motility associated with deregulation of CDC42 activity likely accounts for the modest increase of neutrophil recruitment into peritoneal cavities of thioglycollate-injected mice. In addition, our study suggests that CDC42 mediates neutrophil motility via a specialized adhesion structure related to podosomes, which is regulated by ERK ${ }^{\mathrm{MAPK}}$ downstream of integrins in combination with chemokine receptors. Indeed, 
integrin engagement by fibrinogen led to increased CDC42GAPdeficient neutrophil motility and alteration in podosome-like structures. Finally, ERK activity in CDC42GAP-deficient neutrophils was enhanced by integrin ligation and fMLP stimulation, but not by fMLP alone, which correlated with the increased motility and altered podosome-like structures in the same cells. Podosomes, which are characterized by a conical F-actin core surrounded by a ring of vinculin, are found in highly motile cells, ${ }^{31}$ in nontransformed cells such as monocytes, dendritic cells, macrophages, and osteoclasts, and in neutrophils ${ }^{32,33}$ and eosinophils. ${ }^{34}$ Podosomes are known for their role in matrix bone degradation. However, since they are linked to the extracellular matrix via integrins, podosomes are also thought to establish dynamic contacts necessary for effective cell migration. ${ }^{35}$ The key regulator of podosomes is the product of the gene mutated in WASp, ${ }^{36}$ a specific CDC42 effector. In macrophages and dendritic cells, loss of WASp results in defects in podosome assembly and motility. ${ }^{36-39}$ In our study, clusters and rings of podosome-like structures were present at the leading edge of WT cells. The number of cells displaying this structure was low, which may be due to their short life and dynamic phenotype. ${ }^{31}$ Podosome-like structures were defective in CDC42GAPdeficient cells. However, the apparent loss of podosomes may result from a higher podosome turnover. Indeed, CDC42GAPdeficient cells demonstrated increased motility, and motility is directly correlated with adhesion turnover. In addition, the increased motility and altered podosomes were likely due to sustained ERK activity. ERK localizes at focal adhesions and promotes motility by regulating focal adhesion turnover. ${ }^{24-27,40}$ We were unable to detect phosphorylated ERK in podosomes by immunofluorescence (not shown). However, like focal adhesions, ERK can localize to podosomes via integrin ligation and contributes to osteoclast motility. ${ }^{41}$ Finally, a complex including betaPIX, SPIN90, Nck, and WASp that regulates stable adhesion can be dynamically modulated by adhesion-dependent ERK activity. ${ }^{42}$ Thus, we hypothesize that CDC42 activity via CDC42GAP may regulate neutrophil motility via ERK-induced podosome turnover at the cell's leading edge.

In this study, we also suggest that CDC42 plays an important role in chemotaxis by restraining membrane extension. We report that alteration in $\mathrm{CDC} 42$ activity led to increased membrane protrusions and filopodia-like formations around the cell. Our study is consistent with the view that CDC42 is required to properly orient the cytoskeleton responses toward the chemoattractant. ${ }^{18,43}$ Using cells deficient in the CDC42 GEF Pix $\alpha$, CDC42 has been shown to form a complex with PIX $\alpha$ and PAK and to regulate the intracellular localization of Akt (at the front) and of PTEN (at the back) necessary to polarize F-actin in the gradient of stimulation, and therefore sense the gradient. ${ }^{43}$ Thus, CDC42 is thought to be part of the "chemical compass" during chemotaxis to make the cell front distinct from the back. ${ }^{44}$ The "chemical compass" allows the formation of a leading edge of F-actin by the cooperation of Pi3K signals ${ }^{45,46}$ and the CDC42 pathway, ${ }^{43}$ while inhibitory signals, including PTEN ${ }^{43,47}$ and myosin II, ${ }^{48,49}$ negatively regulate actin polymerization and membrane protrusions in areas outside the leading edge. ${ }^{49}$ In our study, we suggest that CDC42 also regulates chemotaxis via $338^{\mathrm{MAPK}}$, which serves as an inhibitory signal of membrane extensions during chemotaxis. Indeed, p38 $38^{\mathrm{MAPK}}$ was reduced in $\mathrm{CDC}_{2} \mathrm{GAP}^{-1-}$ cells and correlated with the defective chemotactic responses and increased filopodia. Thus, the CDC42-mediated $\mathrm{p} 38^{\mathrm{MAPK}}$ pathway may contribute to inhibitory signals by restraining filopodia to the leading edge and providing positional information to direct migration. ${ }^{49,50}$ Interestingly, the CDC42-mediated $\mathrm{p} 38^{\mathrm{MAPK}}$ pathway appears downstream of integrin molecules in combination with chemokine receptors, suggesting that CDC42 may integrate signals from both G-coupled chemokine and integrin receptors to regulate the "chemical compass." $\alpha_{4}$ integrin-mediated signals can be polarized and can signal at the back of the cell to limit the activation of Rac and the formation of pseudopodia, thus restraining the formation of pseudopodia to the cell front. ${ }^{51}$ In our study, migration measured by Boyden chambers or fibrinogen-coated transwells may engage various integrins. Fibrinogen mainly but not exclusively binds to CD11b/CD18. The identification of the integrin from which CDC42GAP transduces inhibitory signals would be interesting to investigate further. A role for $\mathrm{p} 38^{\mathrm{MAPK}}$ downstream of tumor necrosis factor $\alpha(\mathrm{TNF} \alpha)$ in neutrophil migration by antagonizing filopodia was previously described. This effect appeared to be independent from CDC42, ${ }^{28}$ suggesting that CDC42-mediated cell migration is agonist specific. Alternatively, since CDC42GAP possesses multiple signaling domains in addition to the GAP domain, we cannot fully exclude the possibility that CDC42GAP regulates p38 $8^{\mathrm{MAPK}}$ independently on CDC42. The mechanism by which p38 $8^{\mathrm{MAPK}}$ antagonizes filopodia remains to be established. Whether p38 $8^{\mathrm{MAPK}}$ activity contributes to control $\mathrm{PTEN}^{52}$ or myosin II-related functions in neutrophils would be interesting to investigate further.

The function of CDC42 is likely to be complex. PIX $\alpha$ deficient cells did not show any change in migration rate. The PIX $\alpha / \mathrm{PAK} / \mathrm{CDC} 42$ pathway was described downstream of the G-coupled receptor. ${ }^{19}$ CDC42GAP/CDC42 activity also appears to regulate migration downstream of integrin molecules. Thus, GEFs and GAPs may regulate GTPase activity downstream of specific receptors. ${ }^{53}$

A role for MAPK in cell migration has been largely documented. However, the mechanism by which CDC42 could regulate MAPK activities during neutrophil migration remains to be determined. In fibroblasts or hematopoietic stem cells, alteration of CDC42 activity by CDC42GAP abrogates Janus kinase (JNK) MAPK pathway without impairing either the ERK or the $\mathrm{p} 38^{\mathrm{MAPK}}$ pathway in response to serum or growth factors. ${ }^{21,54}$ This observation reinforces the fact that CDC42 functions may be receptor specific. The CDC42 effectors, WASp and/or PAK, have been largely implicated in podosome assembly and/or adhesion complex turnover. WASp also plays a role in preventing lateral membrane protrusions. ${ }^{55} \mathrm{P} 38^{\mathrm{MAPK}}$ or ERK can be activated downstream of WASp/WAVE proteins ${ }^{56}$ or PAK. ${ }^{57,58}$ The role of WASp and/or PAK in CDC42-mediated MAPK activity downstream of integrin molecules in neutrophil migration is currently under investigation.

In conclusion, this study provides strong evidence that CDC42 activity is important for both random movement and directed migration. Our study highlights the physiologic relevance of CDC42 activity in neutrophil motility since alteration of CDC42 activity led to an overall increased, albeit modest, in vivo neutrophil recruitment into peritoneal cavities despite a defective directed migration. These data reinforce the importance of having a proper cycle between GTP-bound and GDP-bound CDC42 during neutrophil migration. Therefore, this study provides new insights in CDC42 functions in neutrophils, which could be useful for definingCDC42 as a 
molecular target to generate new therapeutic agents for a broad variety of diseases.

\section{Acknowledgments}

We thank Shelli Homan and Victoria Summey-Harner for animal husbandry. We also thank Amgen (Thousand Oaks, CA) for reagents. We are grateful to Dr Harmut Geiger (Cincinnati, $\mathrm{OH}$ ) for providing us with HUVECs and for his help in the transendothelial assay.

This work was supported by the American Society of Hematology Scholar Award to M.-D.F.

\section{Authorship}

Contributions: K.S., H.X., and S.A. performed research; Y.Z. contributed vital new reagents by providing the CDC42GAP knock-out mouse, analytical tools, and advice on the work; and M.-D.F. designed and performed research and wrote the paper.

Conflict-of-interest disclosure: The authors declare no competing financial interests.

Correspondence: Marie-Dominique Filippi, Division of Experimental Hematology, Cincinnati Children's Hospital, 3333 Burnet Ave, Cincinnati OH 45229; e-mail: marie-dominique.filippi@cchmc.org.

\section{References}

1. Ridley AJ, Schwartz MA, Burridge K, et al. Cell migration: integrating signals from front to back. Science. 2003;302:1704-1709.

2. Raftopoulou M, Hall A. Cell migration: Rho GTPases lead the way. Dev Biol. 2004;265:2332.

3. Bar-Sagi D, Hall A. Ras and Rho GTPases: a family reunion. Cell. 2000;103:227-238.

4. Ridley AJ, Hall A. The small GTP-binding protein rho regulates the assembly of focal adhesions and actin stress fibers in response to growth factors. Cell. 1992;70:389-399.

5. Ridley AJ, Paterson HF, Johnston CL, Diekmann D, Hall A. The small GTP-binding protein rac regulates growth factor-induced membrane ruffling. Cell. 1992;70:401-410.

6. Roberts AW, Kim C, Zhen L, et al. Deficiency of the hematopoietic cell-specific Rho family GTPase Rac2 is characterized by abnormalities in neutrophil function and host defense. Immunity. 1999;10:183-196.

7. Li S, Yamauchi A, Marchal CC, Molitoris JK, Quilliam LA, Dinauer MC. Chemoattractant-stimulated Rac activation in wild-type and Rac2-deficient murine neutrophils: preferential activation of Rac2 and Rac2 gene dosage effect on neutrophil functions. J Immunol. 2002;169:5043-5051.

8. Li B, Yu H, Zheng W, et al. Role of the guanosine triphosphatase Rac2 in T helper 1 cell differentiation. Science. 2000;288:2219-2222.

9. Croker BA, Handman E, Hayball JD, et al. Rac2deficient mice display perturbed T-cell distribution and chemotaxis, but only minor abnormalities in $\mathrm{T}(\mathrm{H}) 1$ responses. Immunol Cell Biol. 2002;80: 231-240.

10. Croker BA, Tarlinton DM, Cluse LA, et al. The Rac2 guanosine triphosphatase regulates $B$ lymphocyte antigen receptor responses and chemotaxis and is required for establishment of $B-1 a$ and marginal zone B lymphocytes. J Immunol. 2002;168:3376-3386.

11. Glogauer M, Marchal CC, Zhu F, et al. Rac1 deletion in mouse neutrophils has selective effects on neutrophil functions. J Immunol. 2003;170:56525657.

12. Gu Y, Filippi MD, Cancelas JA, et al. Hematopoietic cell regulation by Rac1 and Rac2 guanosine triphosphatases. Science. 2003;302:445-449.

13. Walmsley MJ, Ooi SK, Reynolds LF, et al. Critical roles for Rac1 and Rac2 GTPases in B cell development and signaling. Science. 2003;302:459462.

14. Benvenuti F, Hugues S, Walmsley M, et al. Requirement of Rac1 and Rac2 expression by mature dendritic cells for T cell priming. Science. 2004;305:1150-1153.

15. Filippi MD, Harris CE, Meller J, Gu Y, Zheng Y, Williams DA. Localization of Rac2 via the $\mathrm{C}$ terminus and aspartic acid 150 specifies superoxide generation, actin polarity and chemotaxis in neutrophils. Nat Immunol. 2004:5:744-751.
16. Sun CX, Downey GP, Zhu F, Koh AL, Thang H, Glogauer M. Rac1 is the small GTPase responsible for regulating the neutrophil chemotaxis compass. Blood. 2004;104:3758-3765.

17. Allen WE, Zicha D, Ridley AJ, Jones GE. A role for Cdc42 in macrophage chemotaxis. J Cell Biol. 1998;141:1147-1157.

18. Srinivasan S, Wang F, Glavas S, et al. Rac and Cdc42 play distinct roles in regulating $\mathrm{PI}(3,4,5) \mathrm{P} 3$ and polarity during neutrophil chemotaxis. J Cell Biol. 2003;160:375-385.

19. Li Z, Hannigan M, Mo Z, et al. Directional sensing requires $\mathrm{G}$ beta gamma-mediated PAK1 and PIX alpha-dependent activation of Cdc42. Cell. 2003; 114:215-227.

20. Symons M, Derry JM, Karlak B, et al. WiskottAldrich syndrome protein, a novel effector for the GTPase CDC42Hs, is implicated in actin polymerization. Cell. 1996;84:723-734.

21. Wang L, Yang L, Filippi MD, Williams DA, Zheng Y. Genetic deletion of Cdc42GAP reveals a role of Cdc42 in erythropoiesis and hematopoietic stem/ progenitor cell survival, adhesion, and engraftment. Blood. 2006;107:98-105.

22. Ding ZM, Babensee JE, Simon SI, et al. Relative contribution of LFA-1 and Mac-1 to neutrophil adhesion and migration. J Immunol. 1999;163: 5029-5038.

23. Huang $\mathrm{C}$, Jacobson $\mathrm{K}$, Schaller MD. MAP kinases and cell migration. J Cell Sci. 2004;117: 4619-4628.

24. Ren XD, Kiosses WB, Sieg DJ, Otey CA Schlaepfer DD, Schwartz MA. Focal adhesion kinase suppresses Rho activity to promote focal adhesion turnover. J Cell Sci. 2000;113 (Pt 20): 3673-3678.

25. Sahai E, Olson MF, Marshall CJ. Cross-talk between Ras and Rho signalling pathways in transformation favours proliferation and increased motility. EMBO J. 2001;20:755-766.

26. Pawlak G, Helfman DM. MEK mediates v-Srcinduced disruption of the actin cytoskeleton via inactivation of the Rho-ROCK-LIM kinase pathway. J Biol Chem. 2002;277:26927-26933.

27. Webb DJ, Donais K, Whitmore LA, et al. FAK-Src signalling through paxillin, ERK and MLCK regulates adhesion disassembly. Nat Cell Biol. 2004; 6:154-161.

28. Gadea G, Roger L, Anguille C, de Toledo M, Gire $V$, Roux P. TNFalpha induces sequential activation of Cdc42- and p38/p53-dependent pathways that antagonistically regulate filopodia formation. J Cell Sci. 2004;117:6355-6364.

29. Keely PJ, Westwick JK, Whitehead IP, Der CJ, Parise LV. Cdc42 and Rac1 induce integrin-mediated cell motility and invasiveness through PI(3)K. Nature. 1997;390:632-636.

30. Mataraza JM, Briggs MW, Li Z, Entwistle A, Ridley AJ, Sacks DB. IQGAP1 promotes cell motility and invasion. J Biol Chem. 2003;278:4123741245 .
31. Linder S, Aepfelbacher M. Podosomes: adhesion hot-spots of invasive cells. Trends Cell Biol. 2003; 13:376-385

32. Jones SL, Brown EJ. FcgammaRII-mediated adhesion and phagocytosis induce L-plastin phosphorylation in human neutrophils. J Biol Chem. 1996;271:14623-14630.

33. Fuortes M, Melchior M, Han H, Lyon GJ, Nathan C. Role of the tyrosine kinase pyk2 in the integrindependent activation of human neutrophils by TNF. J Clin Invest. 1999;104:327-335.

34. Johansson MW, Lye MH, Barthel SR, Duffy AK, Annis DS, Mosher DF. Eosinophils adhere to vascular cell adhesion molecule- 1 via podosomes. Am J Respir Cell Mol Biol. 2004;31:413-422.

35. Burns S, Hardy SJ, Buddle J, Yong KL, Jones GE, Thrasher AJ. Maturation of DC is associated with changes in motile characteristics and adherence. Cell Motil Cytoskeleton. 2004;57:118-132.

36. Calle Y, Chou HC, Thrasher AJ, Jones GE. Wiskott-Aldrich syndrome protein and the cytoskeletal dynamics of dendritic cells. J Pathol. 2004; 204:460-469.

37. Linder S, Nelson D, Weiss M, Aepfelbacher M. Wiskott-Aldrich syndrome protein regulates podosomes in primary human macrophages. Proc Natl Acad Sci U S A. 1999;96:9648-9653.

38. Calle $\mathrm{Y}$, Jones GE, Jagger $\mathrm{C}$, et al. WASp deficiency in mice results in failure to form osteoclast sealing zones and defects in bone resorption. Blood. 2004;103:3552-3561.

39. Snapper SB, Meelu P, Nguyen D, et al. WASP deficiency leads to global defects of directed leukocyte migration in vitro and in vivo. $\mathrm{J}$ Leukoc Biol. 2005;77:993-998.

40. Silletti S, Yebra M, Perez B, Cirulli V, McMahon $\mathrm{M}$, Montgomery AM. Extracellular signal-regulated kinase (ERK)-dependent gene expression contributes to L1 cell adhesion molecule-dependent motility and invasion. J Biol Chem. 2004; 279:28880-28888.

41. Rucci N, DiGiacinto C, Orru L, Millimaggi D, Baron R, Teti A. A novel protein kinase $C$ alphadependent signal to ERK1/2 activated by alphaVbeta3 integrin in osteoclasts and in Chinese hamster ovary (CHO) cells. J Cell Sci. 2005;118:32633275.

42. Lim CS, Kim SH, Jung JG, Kim JK, Song WK. Regulation of SPIN90 phosphorylation and interaction with Nck by ERK and cell adhesion. J Biol Chem. 2003;278:52116-52123.

43. Li F, Higgs HN. The mouse Formin mDia1 is a potent actin nucleation factor regulated by autoinhibition. Curr Biol. 2003;13:1335-1340.

44. Bourne HR, Weiner O. A chemical compass. Nature. 2002;419:21.

45. Weiner OD, Neilsen PO, Prestwich GD, Kirschne MW, Cantley LC, Bourne HR. A PtdlnsP(3)- and Rho GTPase-mediated positive feedback loop regulates neutrophil polarity. Nat Cell Biol. 2002 4:509-513. 
46. Wang F, Herzmark P, Weiner OD, Srinivasan S, Servant G, Bourne HR. Lipid products of $\mathrm{PI}(3) \mathrm{Ks}$ maintain persistent cell polarity and directed motility in neutrophils. Nat Cell Biol. 2002;4:513-518.

47. Li Z, Dong X, Wang Z, et al. Regulation of PTEN by Rho small GTPases. Nat Cell Biol. 2005;7: 399-404.

48. Han YH, Chung CY, Wessels D, et al. Requirement of a vasodilator-stimulated phosphoprotein family member for cell adhesion, the formation of filopodia, and chemotaxis in dictyostelium. J Biol Chem. 2002;277:49877-49887.

49. Affolter M, Weijer CJ. Signaling to cytoskeletal dynamics during chemotaxis. Dev Cell. 2005;9: 19-34.

50. Heid PJ, Geiger J, Wessels D, Voss E, Soll DR. Computer-assisted analysis of filopod formation and the role of myosin II heavy chain phosphorylation in Dictyostelium. J Cell Sci. 2005;118:2225-2237.
51. Nishiya N, Kiosses WB, Han J, Ginsberg MH. An alpha4 integrin-paxillin-Arf-GAP complex restricts Rac activation to the leading edge of migrating cells. Nat Cell Biol. 2005;7:343-352.

52. Wu Y, Hannigan MO, Kotlyarov A, Gaestel M, Wu $D$, Huang CK. A requirement of MAPKAPK2 in the uropod localization of PTEN during FMLPinduced neutrophil chemotaxis. Biochem Biophys Res Commun. 2004;316:666-672.

53. Hall AB, Gakidis MA, Glogauer M, et al. Requirements for Vav guanine nucleotide exchange factors and Rho GTPases in FcgammaR- and complement-mediated phagocytosis. Immunity. 2006;24:305-316.

54. Wang L, Yang L, Burns K, Kuan CY, Zheng Y. Cdc42GAP regulates c-Jun $\mathrm{N}$-terminal kinase (JNK)-mediated apoptosis and cell number during mammalian perinatal growth. Proc Natl Acad Sci U S A. 2005;102:13484-13489.
55. Myers SA, Han JW, Lee Y, Firtel RA, Chung CY. A Dictyostelium homologue of WASP is required for polarized F-actin assembly during chemotaxis. Mol Biol Cell. 2005;16:2191-2206.

56. Sossey-Alaoui K, Ranalli TA, Li X, Bakin AV, Cowell JK. WAVE3 promotes cell motility and invasion through the regulation of MMP-1, MMP-3, and MMP-9 expression. Exp Cell Res. 2005;308:135145.

57. Lee J, Jung ID, Chang WK, et al. p85 beta-PIX is required for cell motility through phosphorylations of focal adhesion kinase and p38 MAP kinase. Exp Cell Res. 2005;307:315-328.

58. Eblen ST, Slack-Davis JK, Tarcsafalvi A, Parsons JT, Weber MJ, Catling AD. Mitogen-activated protein kinase feedback phosphorylation regulates MEK1 complex formation and activation during cellular adhesion. Mol Cell Biol. 2004;24:2308-2317. 